\title{
Clip and wire localization of locally advanced malignant breast masses in patients undergoing neoadjuvant chemotherapy and breast conservation therapy
}

\author{
Lamiaa Adel Salah El Din Shalaby ${ }^{*^{*}}$ D, Emad Salah el din Khallaf ${ }^{2}$ and Mohamed Mahmoud Moussa ${ }^{3}$
}

\begin{abstract}
Background: In locally advanced breast cancers, the use of preoperative systemic therapy has been shown to induce tumor response and to improve the local control rate after subsequent surgery and radiation therapy. The purpose of the study is to evaluate the accuracy of localization of breast malignant masses in patients who received neoadjuvant chemotherapy and will undergo conservative breast surgery by using clip and wire marker.

Results: Clip placement was done in 20/20 cases (100\%). There was no mammographic evidence of clip migration or complication related to the clip insertion. Wire localization of tumor bed marked by radiopaque clip 1-day preoperative was done in 18/20 patient (90\%), the other 2 patients, the masses were clinically palpable (10\%). Accurate localization by wire was positive in 18/18 cases. Clip and wire retrieval were positive in all cases.

Conclusion: Clip markers can be used for tumor localization in breast cancer patients undergoing neoadjuvant chemotherapy without migration. Clips are tolerated and safe for the patient, easily visualized on imaging, do not interfere with treatment response, and are cost-effective. Also, serves as a guide for post-neoadjuvant chemotherapy localization when the tumor is not palpable.
\end{abstract}

Keywords: Clip, Wire, Locally advanced breast cancer, Neoadjuvant chemotherapy, Conservative breast surgery

\section{Background}

Breast cancer is currently the second most common cancer worldwide and, by far, the most frequent malignant tumor among women and female cancer patients $[1,2]$.

Mastectomy followed by postmastectomy radiation has been the standard of treatment for patients with locally advanced breast cancer (LABC) in that a breast-conserving approach for patients with larger primary tumors may not have been technically feasible, may not have been as effective, and may have resulted in significant cosmetic deformity. However, since the introduction of neoadjuvant chemotherapy (NAC), a large number of patients

\footnotetext{
* Correspondence: lamiaadel73@kasralainy.edu.eg

${ }^{1}$ Radiology Department (Women's Imaging Unit), Faculty of Medicine, Kasr

ElAiny Hospital, Cairo University, Cairo, Egypt

Full list of author information is available at the end of the article
}

with locally advanced primary disease have enjoyed a conservative approach [3].

Neoadjuvant chemotherapy (NAC) has been the accepted standard of care for patients with operable or inoperable breast cancers. The benefits of NAC performed prior to surgery are as follows: (1) reduction of mortality; (2) improvement of surgical options, such as conversion to breast-conserving surgery (BCS) in operable patients, as well as surgery in previously inoperable patients; and (3) early collection of information on the treatment response and tumor biology of the breast cancer $[4,5]$.

The combination of neoadjuvant chemotherapy and surgery offers better local disease control and overall survival than surgery alone in patients with breast cancer, especially those with locally advanced breast cancer [6]. 


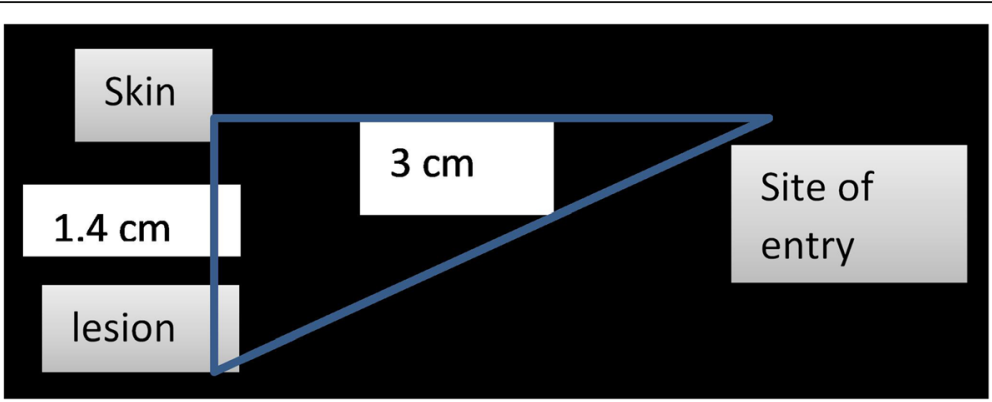

Fig. 1 Diagrammatic illustration explaining the site of entry of wire, its direction, the distance between it and the lesion, the distance between lesion and skin and, lastly the distance between the site of entry and skin

As new chemotherapeutic agents have been developed, patients who have undergone NAC have shown a positive response (approximately 80\%). Sometimes, a dramatic pathologic complete response (pCR) can be achieved. For these reasons, international breast cancer specialist panels in 2006 and 2010 referred to the importance of the radiopaque clip [6]. The placement of radiopaque markers is essential for patients with NAC and BCS because a dramatic $\mathrm{pCR}$ in a patient with a nonradiopaque marker would not allow the surgeon to accurately locate and excise any residual cancerous tissue, or reconstruct the breast with a satisfactory cosmetic result [4].

It was reported that in $47 \%$ of the cases, the metallic markers implanted under sonographic guidance were the only remaining evidence of the original site of the tumor. They conclude that this technique effectively addresses the problem of preoperative localization of the tumor bed in patients who are expected to achieve a complete or near-complete response to NAC [7].

\section{Methods}

\section{Patients}

The study was approved by the hospital ethical committee and informed consent was obtained from all patients. The study included 20 patients presenting with breast malignant masses referred from a multidisciplinary team ( MDT) to the Diagnostic Radiology Department for evaluation during the period from October 2016 to March 2018.

All patients had pathologically proven locally advanced breast cancer, categorized as BIRADS VI, scheduled to receive neoadjuvant chemotherapy with later planning breast-conservative surgery through localization by clip placement and wire localization.

All patients received neoadjuvant chemotherapy. Tumor response was assessed after two cycles of chemotherapy.

\section{- Inclusion criteria:}

If lesion decreases by $50 \%$ of its size or reaches $2 \mathrm{~cm}$ maximum in diameter as the possibility of complete disappearance is anticipated.

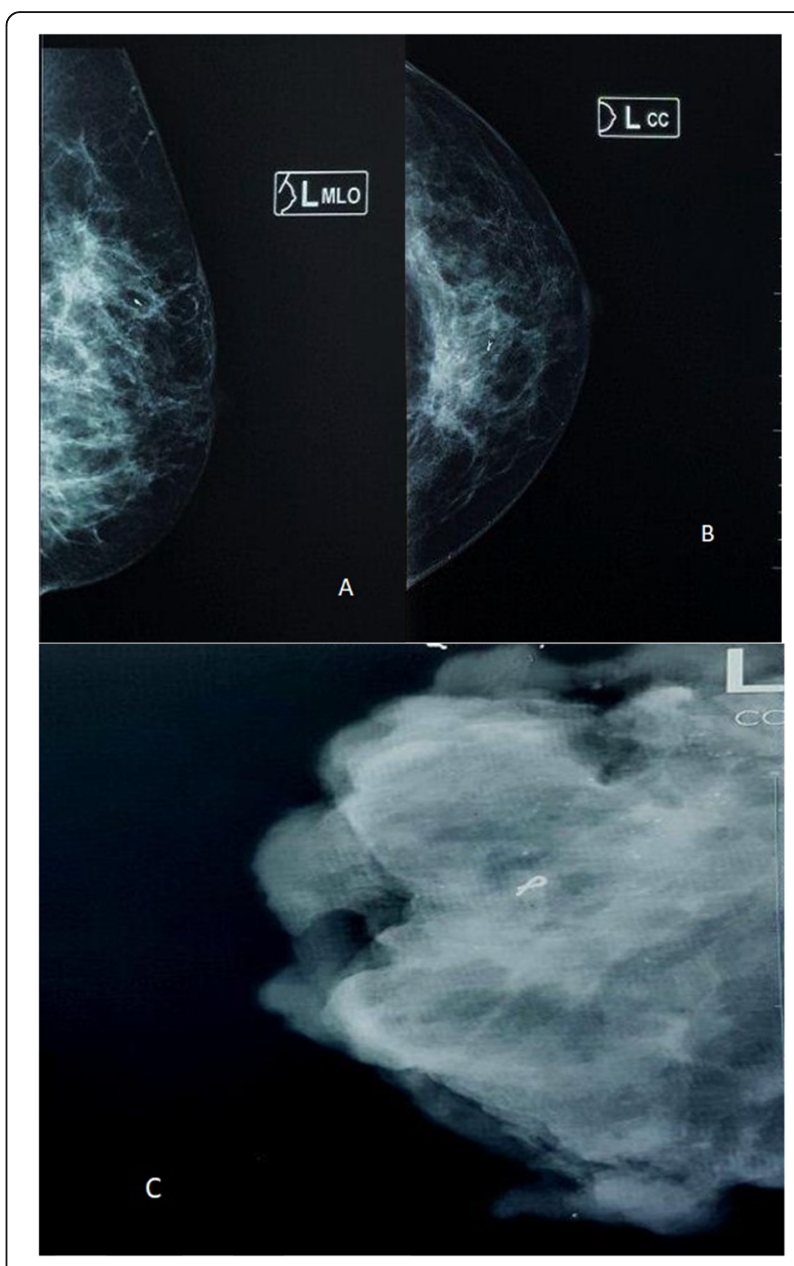

Fig. 2 A 55-year-old female presented with left breast lump proved to be IDC grade I. Left breast MLO $\mathbf{a}$ and CC $\mathbf{b}$ view show UIQ clip. No wire localization was done as the mass was palpable. Intraoperative specimen mammography c show clip retrieved 
Table 1 Preoperative wire localization number and percentage

\begin{tabular}{lll}
\hline & Frequency & Percent \\
\hline Negative & 2 & 10.0 \\
Positive & 18 & 90.0 \\
Total & 20 & 100.0 \\
\hline
\end{tabular}

\section{- Exclusion criteria:}

If lesion has a poor response to NAC and slight or no decrease in size detected

\section{Methods and image analysis}

In all cases (20/20), breast cancer was diagnosed using trucut or core biopsy performed under clinical or sonographic guidance and final histopathological results from the biopsy were reviewed and pathological types of breast cancer and immunochemical markers, including the estrogen receptor, progesterone receptor, and human epidermal growth factor receptor 2 (HER2) were also evaluated.

All cases were subjected to bilateral digital mammography, high-field superficial ultrasound, and clip placement.

\section{Mammography}

Bilateral mammography included routine craniocaudal and mediolateral oblique views of the breasts. Further magnification and compression views were obtained if necessary. All examinations were performed by radiographic technicians under direct supervision.

Four features were specifically assessed in each mammographic examination for each breast: nodular opacity, microcalcifications, architectural distortion, and asymmetric density. More than one feature could be present in a single breast. Contra-lateral findings suspicious for or highly suggestive of malignancy were sampled for biopsy.

\section{Ultrasound}

All patients were examined by whole-breast sonography in transverse and longitudinal planes with knowledge of clinical and mammographic findings using a high-resolution 5-12 MHz superficial liner small parts transducer. Sometimes, in very large breasts and deep-seated tumors, the 3.5 $\mathrm{MHz}$ curvilinear probe was used and the maximum dimension of the lesion or lesions corresponding to the known malignancy was measured. For the inner medial breast, scanning was performed with the patient in the supine

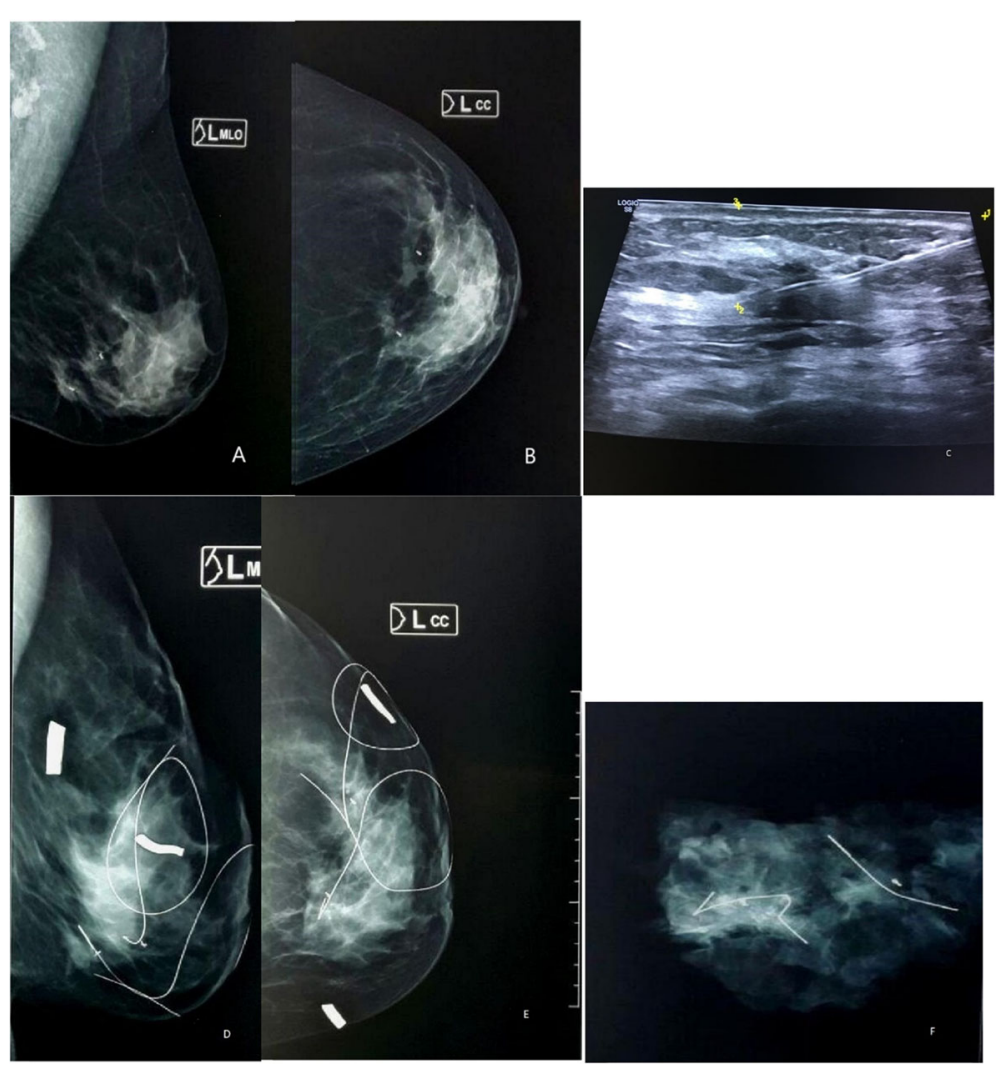

Fig. 3 A 53-year-old female presented with two left breast lumps proven to be IDC grade II. Left breast MLO a and CC $\mathbf{b}$ view show LOQ suspicious spiculated masses with the clips. US-guided clip placement $\mathbf{c}$. Preoperative wire localization MLO d and CC e. Intraoperative specimen mammography $\mathbf{f}$ shows clip and wire retrieved 
position. For the outer lateral breast, the patient was placed in the contralateral oblique position with a pillow under her shoulder and her arm raised. Survey systematic scanning was done in sagittal and transverse planes and in other planes whenever necessary according to the long axis of the mass. Both axillae were evaluated for pathological lymph nodes. Lymph nodes reported as larger than $1 \mathrm{~cm}$, matted, or exhibiting loss of fatty hilum, thickened cortex, or irregular node contour were considered pathologic.

\section{Clip placement}

Commercial disposable clip applier was used for all patients scheduled for NAC. A short skin incision was made using local anesthesia under aseptic conditions. Location of the clips was confirmed by US immediately after clip insertion. The inserted clips appeared as linear hyperechoic structures with or without posterior shadowing. In case of multifocal lesions, additional clip was placed. Postprocedural mammography was performed to confirm objectively the appropriate location of the inserted clips.

\section{Wire localization}

Reassessment by sonomammography is done 1-day preoperatively and wire localization is used in patients with no palpable masses. Breast conservative surgery is done to the patients by the removal of the tumor and surrounding margins of normal breast tissue. With a detailed report written by the radiologist commenting on site of entry of wire, its direction, distance between it and the lesion, the distance between lesion and skin, and lastly, the distance between the site of entry and skin, with illustrative drawing explaining it (Fig. 1).

Postoperative specimen mammography was done for ascertaining removal of wire, clip, mass, and any nearby micro-calcification or architectural distortion if present and assessment of safety margin of removal. Two pathological examinations were done to the patients, first intraoperative frozen section study to examine margins so that in cases margin was infiltrated, re-excision is done and if the margin was free then final paraffin examination of the specimen was done.

\section{Statistics}

Data were statistically described in terms of mean \pm standard deviation $( \pm S D)$, median and range, or frequencies (number of cases) and percentages when appropriate.

\section{Results}

The current study included 20 patients with pathologically proven breast cancer and staged as locally advanced by clinical examination and/or imaging methods.

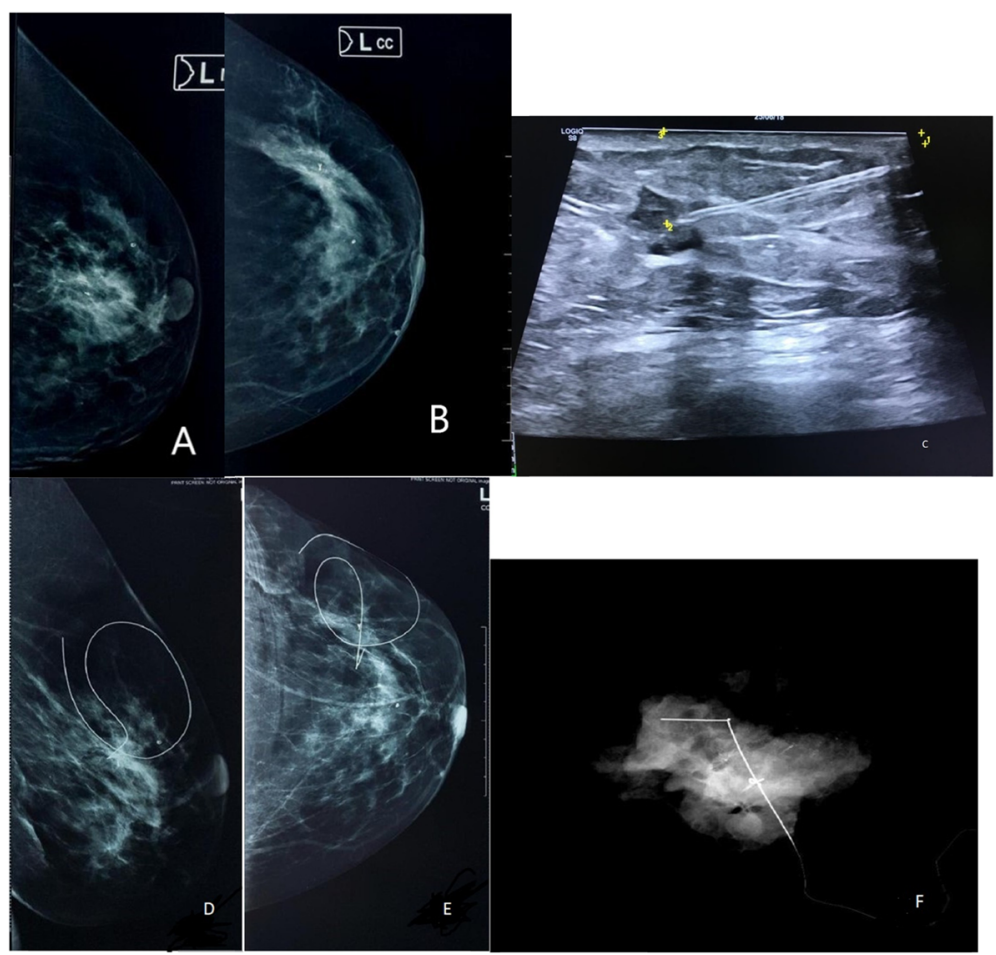

Fig. 4 A 58-year-old female presented with left breast lump proved to be IDC grade II. Left breast MLO $\mathbf{a}$ and CC $\mathbf{b}$ view show UIQ suspicious mass with the clip. US-guided clip placement $\mathbf{c}$. Preoperative wire localization MLO d and CC e. Intraoperative specimen mammography $\mathbf{f}$ shows clip and wire retrieved 
Patients statistically evaluated in the study were pathologically proven malignant, BIRADS VI, with the reference standard being the samples that were obtained with core biopsy, trucut biopsy performed under sonographic guidance, with mean age 46.60 and standard deviation \pm 8.858. The highest frequency of cases $(40 \%)$ occurs between 46 and 55 years.

Clip placement was done to localize the lesion in all the 20 cases $(100 \%)$. There was no mammographic evidence of clip migration during postprocedural follow-up, preoperative final follow-up, and in surgical specimens. Moreover, no complication related to the clip insertion was noted during the study period, and no patient complained of heat sensation or pain.

Localization of tumor bed marked by radiopaque clip using US-guided placement of wire 1-day preoperatively was done in 18 patients $(90 \%)$ and was not done in 2 patients where the masses were clinically palpable (10\%) (Fig. 2), Table 1.

Accurate localization by wire was positive in all the 18 cases as confirmed by ultrasound and the mean distance between wire and clip was $0.36 \mathrm{~cm}$ (Figs. 3, 4, 5, 6 and 7).

The 20/20 patients underwent breast-conservative surgery after completion of neoadjuvant chemotherapy.
Specimen radiological examination was done to assess the clip retrieval inside the specimen, and it was positive in 20 patients $(100 \%)$.

Wire retrieval was positive in all the $18 / 18$ cases who had preoperative wire localization.

Margins were assessed radiologically, frozen section intraoperative and paraffin section postoperative (Fig 8).

- Radiological examination by specimen mammography; margin was free in 20 patients (100\%).

- Intraoperative frozen section examination; margin was free in 19 patients (95.5\%), infiltrated in one patient (5\%).

- Postoperative paraffin section examination; margin was free in 19 patients (95\%), infiltrated in one patient (5\%) and re-excision was done till free margin reached.

The pathology results for the 20 patients showed that 19 (95\%) were invasive duct carcinoma and one (5\%) was invasive duct carcinoma with medullary features Table 2.

The grade of tumor in the 20 patients was grade 1 in 3 (15\%), grade 2 in $15(75 \%)$, and grade 3 in two cases (10\%) Table 3.

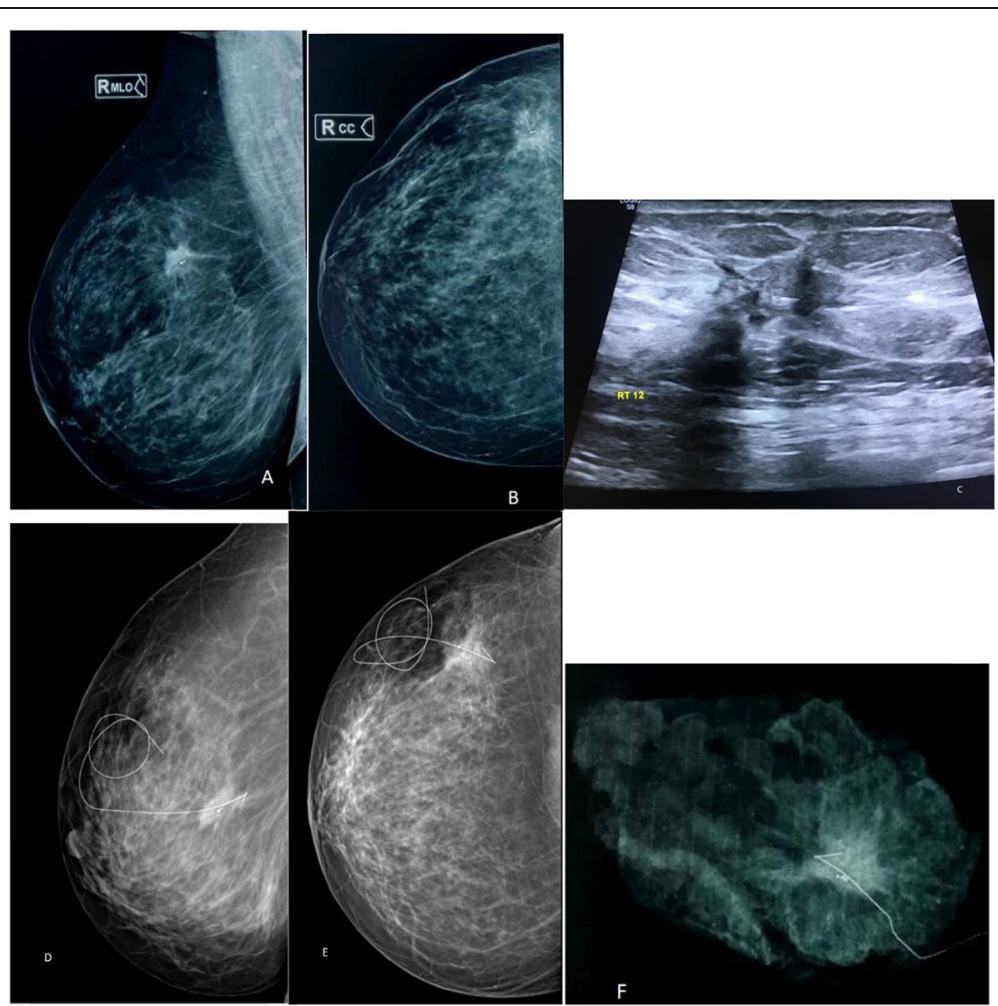

Fig. 5 A 44-year-old female presented with right breast lump proved to be IDC grade II. Right breast MLO a and CC $\mathbf{b}$ view show UOQ spiculated malignant mass with the clip. US-guided clip placement $\mathbf{c}$. Preoperative wire localization MLO $\mathbf{d}$ and CC e. Intraoperative specimen mammography $\mathbf{f}$ shows clip and wire retrieved 


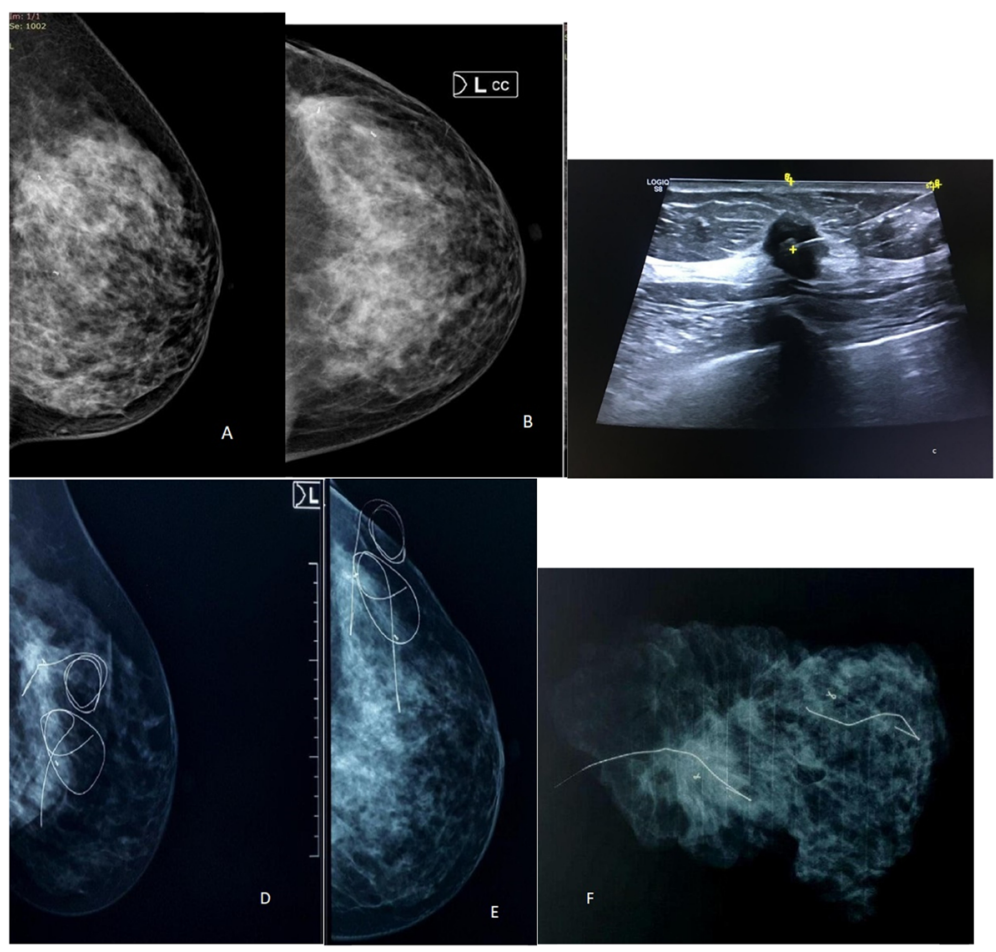

Fig. 6 A 48-year-old female presented with two left breast lump proved to be IDC grade II. Left breast MLO a and CC b view show UIQ suspicious mass with the clip. US-guided clip placement $\mathbf{c}$. Preoperative wire localization MLO $\mathbf{d}$ and CC e. Intraoperative specimen mammography $\mathbf{f}$ shows clip and wire retrieved.

The immunohistopathological examination of hormone receptors of the tumors using ER, PR, Her2, and Ki67 markers showed:

- ER marker was positive in 10 patients (50\%) and negative in 10 patients (50\%)

- PR marker was positive in 11 patient (55\%) and negative in 9 patients (45\%)

- Her2 marker was positive in 11 patient (55\%) and negative in 9 patients (45\%)

- Ki67 marker was high in 16 patients (80\%) and low in 4 patients $(20 \%)$

\section{Discussion}

Breast cancer is the most frequently diagnosed malignancy and the second most common cause of cancerrelated death in women worldwide [8].

Despite recent advances in screening mammography, locally advanced breast cancer remains a challenging clinical problem [9] accounting for $50-70 \%$ of patients with breast cancer presenting for treatment [10].

LABC includes large tumors ( $>5 \mathrm{~cm}$ or T3), tumors of any size that involve skin and/or chest wall (cT4a-c or Stage IIIB), tumors with fixed or matted axillary lymph nodes or tumors clinically detected in the ipsilateral internal mammary nodes without involvement of axillary lymph nodes (N2), and tumors that involve ipsilateral infraclavicular, supraclavicular, or internal mammary lymph nodes with axillary lymph nodes involvement (cN3 or Stage IIIC) [11].

Nineteen out of the 20 patients (95\%) in this study were diagnosed invasive duct carcinoma close to the results of Masroor et al. [12] who stated that $88 \%$ of the cases were invasive duct carcinoma.

Untch et al. [13] stated that although one of the major benefits of neoadjuvant chemotherapy is the possibility to assess the clinical response of the primary tumor and reduction in its size which can range from a minimal response to clinical complete response (cCR), the later effects often make it difficult for surgeons and pathologists to clearly identify the former tumor area and ensure clear resection margins. The insertion of a clip at the time of diagnosis can ensure the identification of the tumor area after multiple cycles of chemotherapy and improve the accuracy of the surgical excision and subsequent pathological assessment after neoadjuvant therapy. Similar to McLaughlin [14], whose study stated that surgeons considering neoadjuvant chemotherapy and the possibility of BCT must ensure the tumor is properly 


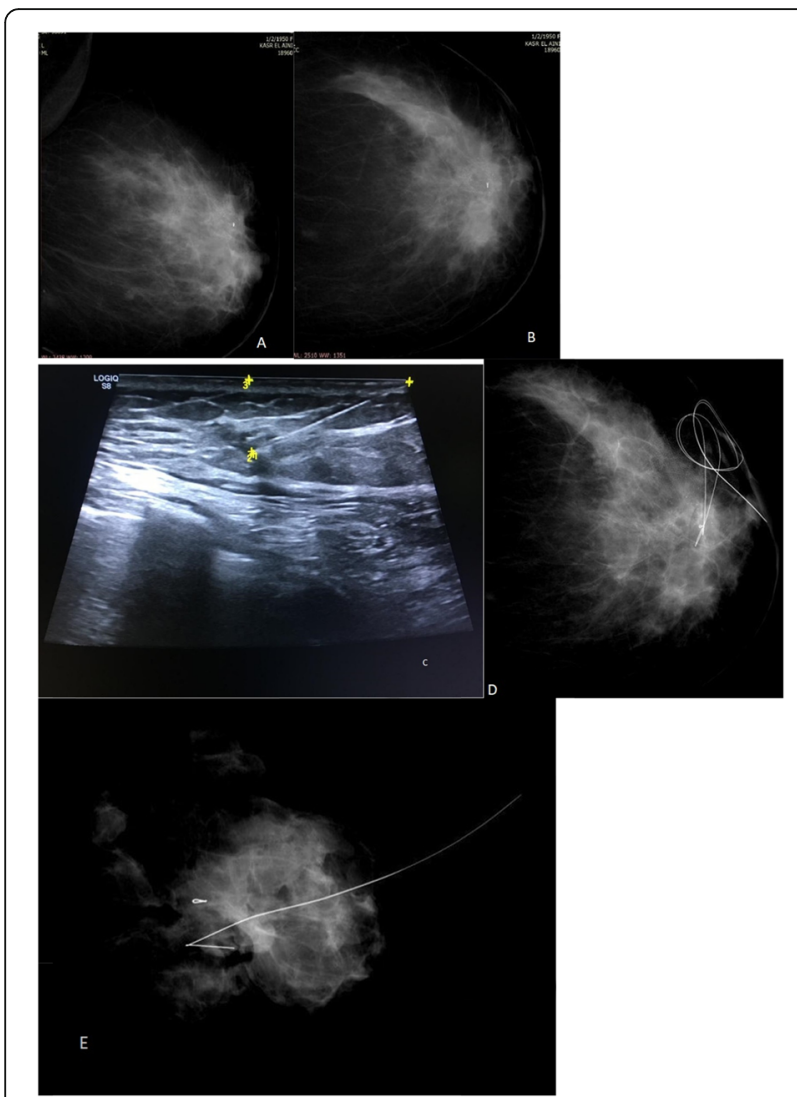

Fig. 7 A 53-year-old female presented with left breast lump proved to be IDC grade III. Left breast MLO $\mathbf{a}$ and CC $\mathbf{b}$ view show UOQ suspicious mass with the clip. US-guided clip placement $\mathbf{c}$. Preoperative wire localization CC view $\mathbf{d}$. Intraoperative specimen mammography e shows clip and wire retrieved

marked with a clip before the start of therapy to allow localization and removal of the tumor bed in the event of a cCR.

The use of a marker clip placed in the tumor bed has been reported as a safe and inexpensive technique that
Table 2 Histopathology diagnosis for all patients

\begin{tabular}{|c|c|c|}
\hline & Frequency & Percent \\
\hline IDC & 19 & 95.0 \\
\hline IDC with medullary features & 1 & 5.0 \\
\hline Total & 20 & 100.0 \\
\hline
\end{tabular}

IDC Invasive ductal carcinoma

allows for subsequent localization of the tumor bed before surgical resection in patients who are receiving neoadjuvant chemotherapy [4].

The current research is a prospective study to evaluate the localization of breast malignant masses in patients who received neoadjuvant chemotherapy and will undergo conservative breast surgery by using clip and wire marker.

In the present study, $40 \%$ of patients $(8 / 20)$ with breast cancer were in the age group from 46 to 55 years in agreement with Siegel et al. [15] study where breast cancer was most common in the age group 40-59 years. However, Mir and Singh [16] reported in their epidemiological study that the highest frequency of breast cancer occurred between 45 and 49 years.

In this study, all patients received neoadjuvant chemotherapy. Tumor response was assessed by comparing the imaging findings after two cycles of chemotherapy. In concordance with $\mathrm{OH}$ et al. [17], clip placement was done if lesion decreased to $50 \%$ of its size or reached 2 $\mathrm{cm}$ in diameter as the possibility of complete disappearance is anticipated.

In our study, wire localization was done on radiopaque clip in 18 cases with non-palpable lesions at the end of neoadjuvant chemotherapy for accurate and easy approach by the surgeon to the tumor bed and in the other 2 patients. The mass was clinically palpable and there was no need for wire localization.

In concordance with our study, Chan et al. [18] and Sharek et al. [19] stated that the most widely adopted

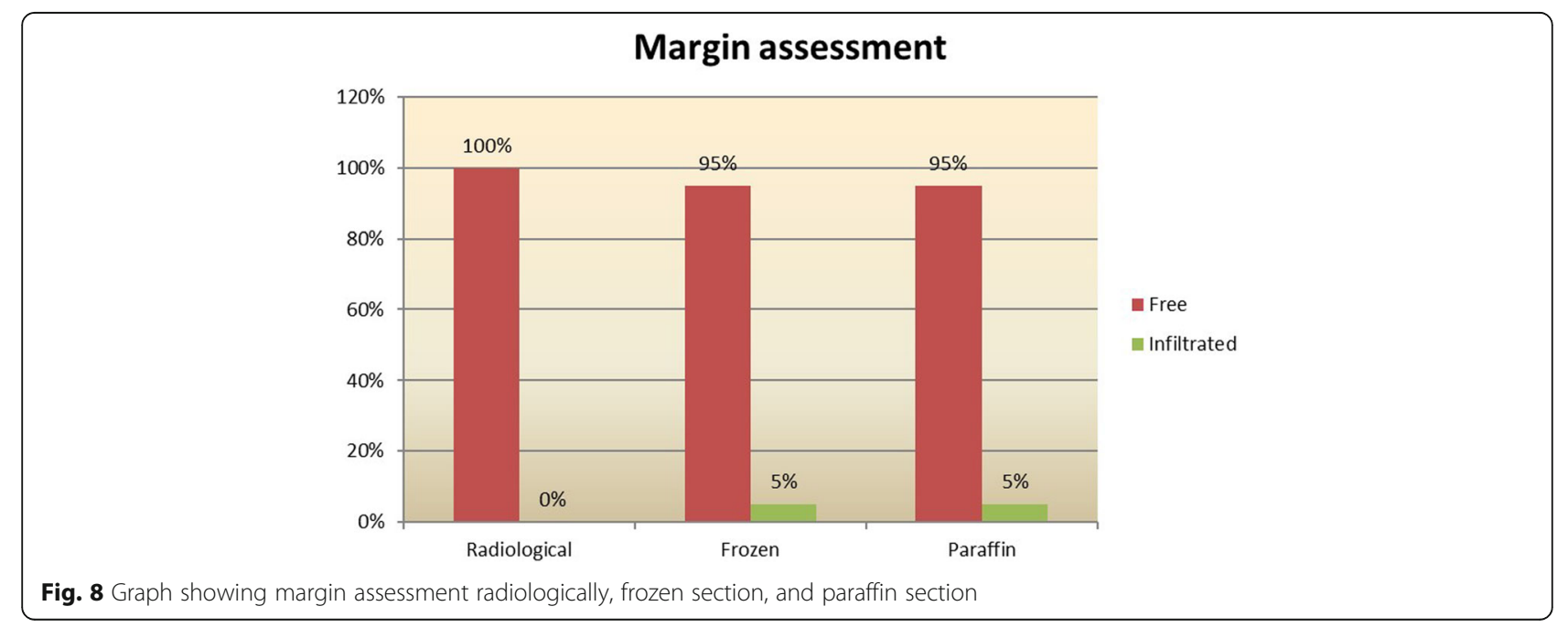


Table 3 The total numbers and percentage of different grades of tumor

\begin{tabular}{lll}
\hline & Frequency & Percent \\
\hline Grade 1 & 3 & 15.0 \\
Grade 2 & 15 & 75.0 \\
Grade 3 & 2 & 10.0 \\
Total & 20 & 100.0 \\
\hline
\end{tabular}

approach radiographic wire localization immediately before surgical resection is currently the accepted standard of care for preoperative localization of nonpalpable breast lesions.

While Ramos et al. [20], reported that wire-guided localization is the standard procedure but is a timeconsuming procedure because it requires the expertise of an experienced radiologist and it is uncomfortable and usually stressful for the patient. Moreover, it may be associated with a high number of positive margins, increases in local recurrences, inadvertent wire displacement during patient transfer, surgical positioning, or during postprocedure mammography and a poor cosmetic result while intraoperative ultrasound (IOUS) excision, an attractive and recommendable practice as an alternative to other excision techniques in patients with complete clinical responses after NACT as wire localization.

In our study, as in studies conducted by Youn et al. [4] and Masroor et al. [12], there was no evidence of clip migration during postprocedural follow-up, preoperative final follow-up, and in surgical specimens. Moreover, no complication related to the clip insertion was noted during the study period, and no patient complained of heat sensation or pain.

Achieving negative surgical margins is a hallmark of successful BCT because this is associated with a lower rate of local recurrence [14].

In the current study, the specimens were examined radiologically by mammography to ensure clip and wire presence and to assess the margin. The clip and wire retrieval was positive in all cases similar to Masroor et al. [12] study. The margin was free radiologically in 17 out of the 18 patients underwent wire localization with accuracy of $95 \%$. These results were close to Ihrai et al. [21], results that stated specimen mammography allowed the achievement of negative margins in $93.5 \%$ of the cases.

\section{Conclusion}

Clip markers can be used for tumor localization in breast cancer patients undergoing NACT without migration. Our results have shown that clips are well tolerated and safe for the patient, easily visualized on imaging, do not interfere with treatment response, and are costeffective. Also, serves as a guide for post-NAC localization when the tumor is not palpable.

\section{Abbreviations}

BCS: Breast-conserving surgery; CCR: Complete response; IDC: Invasive ductal carcinoma; LABC: Locally advanced breast cancer; MDT: Multidisciplinary team; NAC: Neoadjuvant chemotherapy; pCR: Pathologic complete response

\section{Acknowledgements \\ No acknowledgements.}

\section{Authors' contributions}

ASL is the guarantor of the integrity of the entire study. ASL, KES, and MMM contributed to the study concepts and design, literature research, clinical studies, experimental studies/data analysis, and manuscript editing. ASL and MMM contributed to the statistical analysis. ASL and KES contributed to the manuscript preparation. All authors have read and approved the manuscript.

\section{Funding}

No source of funding.

\section{Availability of data and materials}

The corresponding author is responsible for sending the used data and materials upon request.

\section{Ethics approval and consent to participate}

The study was approved by the ethical committee of the oncology, surgery and the Radiology Departments, Faculty of Medicine, Cairo University (Academic highly specialized multidisciplinary Hospital) and an informed written consent was taken from all the patients that were included in the study for performing interventional procedures (clip and wire localization) as a part during their management receiving neoadjuvant chemotherapy and performing conservative breast surgery. If the patient was less than 16 years old, deceased, or unconscious when consent for publication was requested, written informed consent for the publication of this data was given by their parent or legal guardian.

The ethics committee's reference number is not applicable.

The name of the committee in Radiology Department, Cairo University is Ethical committee for research.

\section{Consent for publication}

All authors approved the manuscript. All patients included in this research gave written informed consent to publish the data contained within this study. If the patient was less than 16 years old, deceased, or unconscious when consent for publication was requested, written informed consent for the publication of this data was given by their parent or legal guardian.

\section{Competing interests}

The authors declare that they have no competing interests.

\section{Author details}

${ }^{1}$ Radiology Department (Women's Imaging Unit), Faculty of Medicine, Kasr ElAiny Hospital, Cairo University, Cairo, Egypt. ${ }^{2}$ Department Faculty of Medicine, Kasr Al-Ainy Hospital, Cairo University, El-Manial, Cairo 11956, Egypt. ${ }^{3}$ Oncology Department, Faculty of Medicine, Kasr ElAiny Hospital, Cairo University, Cairo, Egypt.

Received: 21 September 2019 Accepted: 16 October 2019 Published online: 19 December 2019

\section{References}

1. Ferlay J, Soerjomataram I, Diskshit R, et al (2015). Cancer incidence and mortality worldwide: sources, methods and major patterns in GLOBOCAN 2012 Issue International Journal of Cancer International Journal of Cance 136(5):E359-E386

2. Park H, Young M, Kim Z et al (2017). Korean Breast Cancer Society. Basic facts of breast cancer in korea in: the 10-year overall survival progress. J Breast Cancer 20(1):1-11

3. Alm el-din AM, Taghian GA (2009) Breast conservation therapy for patients with locally advanced breast cancer. Semin Radiat Oncol 19:229-235

4. Youn I, Choi SH, Kook SH et al (2015) Ultrasonography-guided surgical clip placement for tumor localization in patients undergoing neoadjuvant chemotherapy for breast cancer.J. Breast Cancer 18(1):44-49 
5. Evangelista L, Cervino AR, Sanco R et al (2014) Use of a portable gamma camera for guiding surgical treatment in locally advanced breast cancer in a post-neoadjuvant therapy setting. Breast Cancer Res Treat:1-10

6. Le-Petross HC, Hylton N (2010) Role of Breast MR Imaging in Neoadjuvant Chemotherapy. Magn Res Imaging Clin North Am 18(2):249-258

7. Bravo EM, Aviles SA, Esgueva A, Cordoba O et al (2011) Breast conservative surgery after neoadjuvant chemotherapy in breast cancer patients: Comparison of two tumor localization methods. EJSO 37:1038-1043

8. Tryfonidis K, Senkus E, Cardoso MJ et al (2015) Management of locally advanced breast cancer-perspectives and future directions. Nat Rev Clin Oncol. 12(3):147-162

9. Whitman GJ, Strom EA (2009) Workup and staging of locally advanced breast cancer. Seminars in Radiation Oncology 19(4):211

10. Reddy SV, Anuradha B, Dinesh N, et al (2016). A Case Study On Epidemiology, Clinico-Pathological, Clinico-Radiological Correlation, Management And Follow Up Of Carcinoma Breast. J. Evid. Based Med. Healthc., pISSN- 2349-2562, elSSN- 2349-2570/ Vol. 3/Issue 31; P. 1475-1477.

11. Chu QD, Adjepong-Tandoh EK and Duda RB (2015). Locally advanced breast cancer (LABC). Surg Oncol 113-140

12. Masroor I, Zeeshan S, Afzal S et al (2015) Outcome and cost effectiveness of ultrasonographically guided surgical clip placement for tumor localization in patients undergoing neo-adjuvant chemotherapy for breast cancer asian pacific. J Cancer Prev 16:8339-8343

13. Untch M, Konecny GE, Paepke S et al (2014) Current and future role of neoadjuvant therapy for breast cancer. Breast:1-12

14. McLaughlin SA (2013) Surgical management of the breast: breast conservation therapy and mastectomy. Surg Clin N Am 93:411-428

15. Siegel RL, Miller KD, Jemal A (2015) Cancer Statistics. Ca Cancer J Clin 65:5-29

16. Mir R, Singh VP (2009) Breast cancer in young women and its impact on reproductive function. Apollo Med 6(3):200-208

17. $J \mathrm{~L} \mathrm{OH}$, Nguyen G, Whitman GJ et al (2007) Placement of radiopaque clips for tumor localization in patients undergoing neoadjuvant chemotherapy and breast conservation therapy. Cancer 110:2420-2427

18. Chan BKY, Wiseberg-Firtell JA, Jois RHS, et al (2015). Localization techniques for guided surgical excision of non-palpable breast lesions. Cochrane Database of Systematic Reviews 2015, Issue 12. Art. No.: CD009206.

19. Sharek D, Zuley ML, Zhang JY et al (2015) Radioactive seed localization versus wire localization for lumpectomies: a comparison of outcomes. AJR 204:870-877

20. Ramos M, Diez JC, Ramos T et al (2014) Intraoperative ultrasound in conservative surgery for non-palpable breast cancer after neoadjuvant chemotherapy. Int J Surg:1-6

21. Ihrai T, Quaranta D, Fouche $Y$ et al (2014) Intraoperative radiological margin assessment in breast-conserving surgery. EJSO 40:449-453

\section{Publisher's Note}

Springer Nature remains neutral with regard to jurisdictional claims in published maps and institutional affiliations.

\section{Submit your manuscript to a SpringerOpen ${ }^{\circ}$ journal and benefit from:}

- Convenient online submission

- Rigorous peer review

- Open access: articles freely available online

- High visibility within the field

- Retaining the copyright to your article

Submit your next manuscript at $\boldsymbol{\nabla}$ springeropen.com 